\title{
Covid-19 Rapid transformation of the medical on-call rotas to deal with the COVID-19 pandemic: a case study
}

\author{
Authors: Amir Aziz, ${ }^{A}$ Neel Patel, ${ }^{B}$ Trishna Chakravorty, ${ }^{C}$ May Yan, ${ }^{D}$ David Gull, , Sarbjit Clare ${ }^{F}$ and Chetan Varma ${ }^{G}$
}

\begin{abstract}
At Sandwell and West Birmingham Hospitals Trust, an emergency rota was put into place in anticipation of the COVID-19 pandemic. Key changes included re-deploying non-general medical (GIM) consultants on to the GIM on-call rota and re-deploying junior doctors on to medical rotas, and introducing a COVID-19 induction training programme to support these redeployments. Results from a survey showed $100 \%$ of consultants felt the rotas were resilient, with $96 \%$ of consultants stating they felt the rotas were well-staffed and $77 \%$ stating that they observed no drop in quality of care. Here we outline how these changes were made and present quantitative and qualitative feedback, with the aim of informing other trusts carrying out similar urgent reconfigurations in the future, or seeking to apply the lessons learnt to their non-emergency rotas.
\end{abstract}

KEYWORDS: Medical on-call rotas, workforce planning, COVID-19

DOI: $10.7861 /$ fhj.2020-0148

\section{Introduction}

A pandemic due to COVID-19 was declared on 11 March 2020 by the World Health Organization' and has led to immense pressure on healthcare services. Sandwell and West Birmingham Hospitals Trust (SWBH) consists of two teaching hospitals, City Hospital and Sandwell General Hospital, which deliver care to a population of 600,000 people. On average, $80-100$ medical patients are admitted each day.

Authors: ${ }^{A}$ Cardiology fellow, Sandwell and West Birmingham NHS Trust; ${ }^{B}$ Consultant in acute medicine, Sandwell and West Birmingham NHS Trust; ' ${ }^{C}$ consultant in acute medicine, Sandwell and West Birmingham NHS Trust, and deputy training programme director for acute internal medicine, Health Education England West Midlands; ${ }^{D}$ consultant in acute medicine, Sandwell and West Birmingham NHS Trust, and deputy training programme director CMT/IMT, Health Education England West Midlands; ${ }^{E}$ Consultant in stroke medicine, Sandwell and West Birmingham NHS Trust; ${ }^{F}$ consultant in acute medicine and deputy medical director, Sandwell and West Birmingham NHS Trust; ${ }^{6}$ Consultant interventional cardiologist, Sandwell and West Birmingham NHS Trust
An emergency rota was put into place in anticipation of the first wave of COVID-19 cases. The challenge was to balance the following requirements:

> Managing the increase in admissions and maintaining a greater ward presence over a longer period.

> Separating COVID-19 positive/suspected patients and nonCOVID patients on both sites.

> Ensuring staff had the right skill-mix and were effectively training and supported.

> Having resilience to cover staff sickness.

$>$ Ensuring smooth running of inpatient care and minimising delays to patient pathways by maintaining 7-day working.

> Complying with European Working Time directives.

We present in detail what was required and how we changed rotas for each grade of doctor.

\section{Methodology}

In response to COVID-19 the Group of Medicine made the following key changes:

> Identifying consultants who had general medical (GIM) duties but were not on the GIM on-call rota (those working in specialties such as stroke and gastroenterology, and ward locums) to increase availability for a consultant rota.

> Partnering consultants with GIM training but who were no longer involved in GIM (those working in specialties such as cardiology, rheumatology and dermatology) with consultants currently participating in GIM for support purposes.

> Moving junior doctors from non-medical specialties to medical rotas, again partnering them with those with GIM experience for support.

> Creating a new bespoke respiratory rota of all grades to support a large new unit for non-invasive ventilation (NIV) and continuous positive airway pressure (CPAP).

A COVID-19 training programme was implemented for medical colleagues at all levels. This web-based programme, which covered topics from advanced life support in COVID-19 to escalation and end-of-life discussions, ensured that medical staff had the knowledge and skills necessary to deal with COVID-related issues and the acute medical take. As the programme was remote it was also rapidly taken up by other local Trusts. 

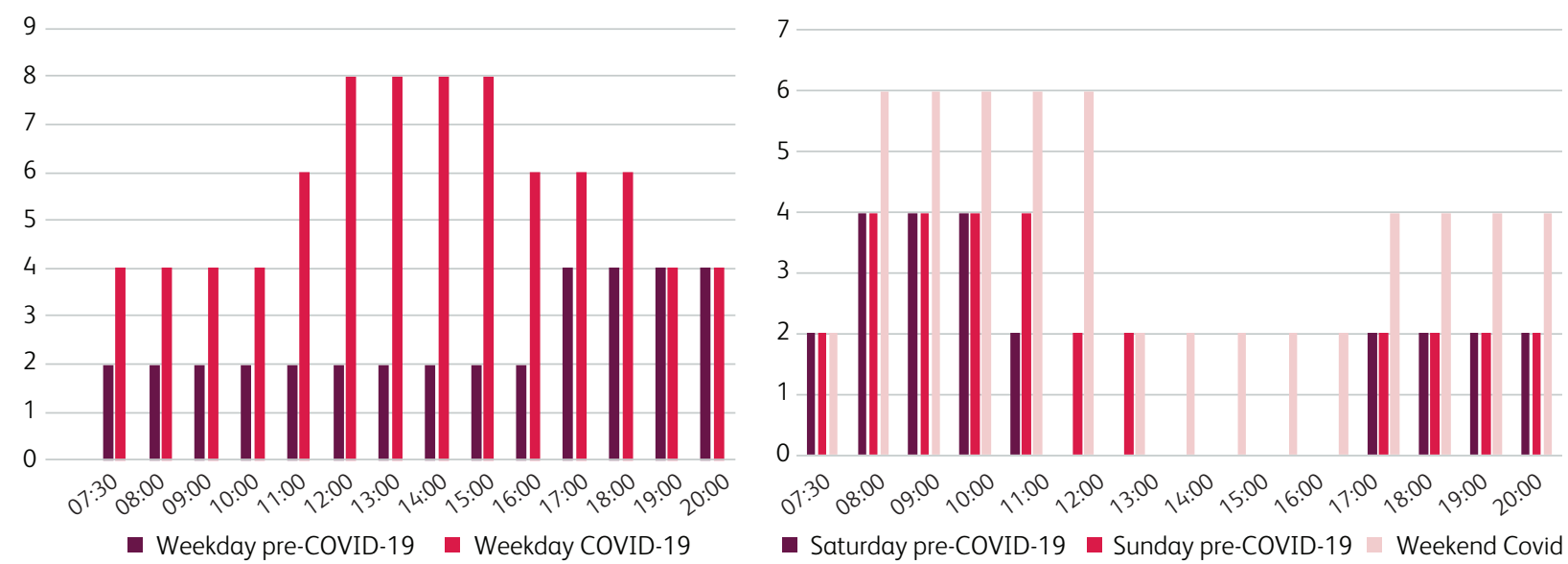

Fig 1. Comparison of consultant presence by hour and the number of consultants on the original rota and the COVID-19 rota during (a) weekdays and (b) weekends.

\section{Consultant rota and ward cover (30 March 2020 - 15 June 2020)}

The traditional model of acute medical inpatient care was a single acute medical take at each site. The first step was to divide the acute medical take into two separate medical takes at each site: patients with confirmed or suspected COVID-19 and non-COVID patients. The challenge was to create teams where the COVID take could be prioritised and staff redeployed in case of admission surges.

The consultant on call-rota was expanded from 26 to 48 consultants: nine acute medicine physicians, three gastroenterologists and two stroke physicians who were not on the GIM rota were redeployed; six locum consultants were recruited; and any physicians working part-time were moved to a full-time slot, working one in six weekends. Two to six extra consultants were working throughout the day at any one time, with continuous on-site presence on the weekends; this differed from the original on-call rota (Fig 1). A second consultant ward cover was introduced from specialties that were not active in GIM but had a GIM background, including dermatology, rheumatology, genito-urinary medicine and neurology. These consultants were partnered with ward consultants in GIM medicine to gain experience. This allowed the GIM ward consultants to have breaks during the week and if there was absence due to sickness, self-isolation or shielding there was a level of cover to allow continuity of care.

Rota for medical registrars (6 April 2020 - 4 August 2020)

Medical registrars (ST3 and above) are core to the functioning of the medical take and wider on-call service. To help with 'buy-in' and engagement from the registrars, one key step was to involve a senior registrar (registrar forum lead) to lead and help deliver the changes necessary.

The new on-call medical team was expanded from 11 to 21 registrars, divided into two separate on-call rotas - a 1 in 14 COVID rota which included experienced GIM registrars and a 1 in 7 non-COVID rota which included medical registrars not on the GIM rota (see Fig 2). This involved the following changes:
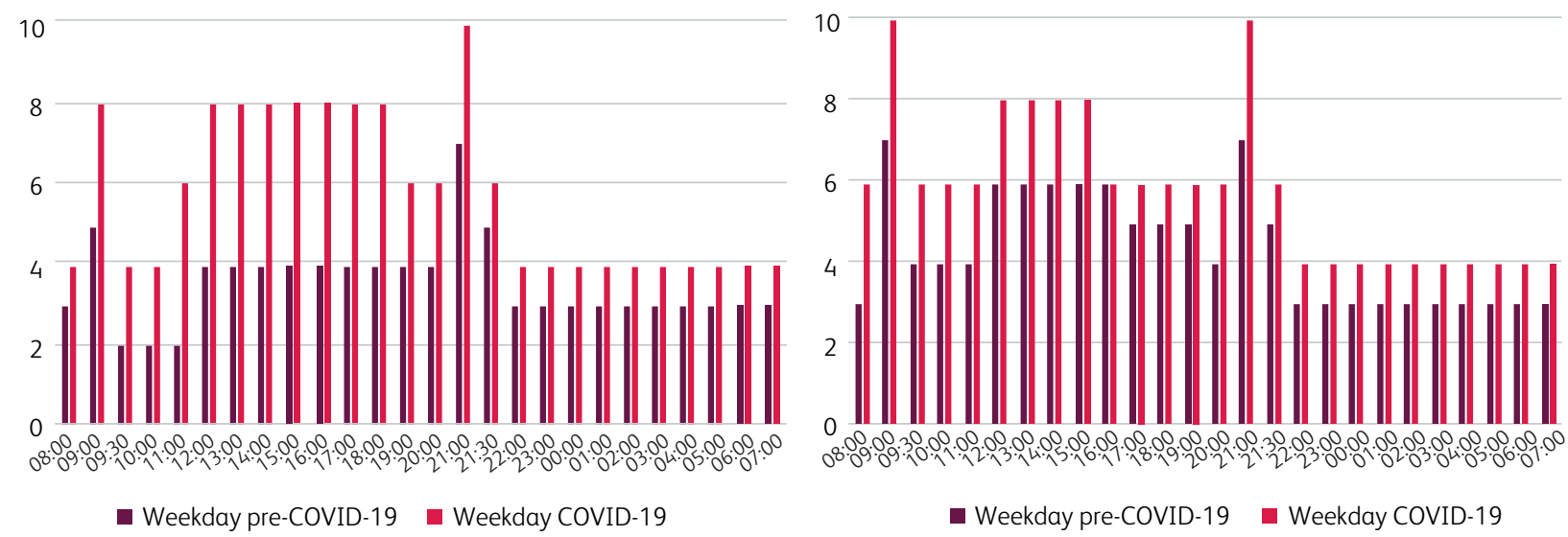

Fig 2. Comparison of registrar presence by hour and the number of registrars on the original rota and the COVID-19 rota during (a) weekdays and (b) weekends. 

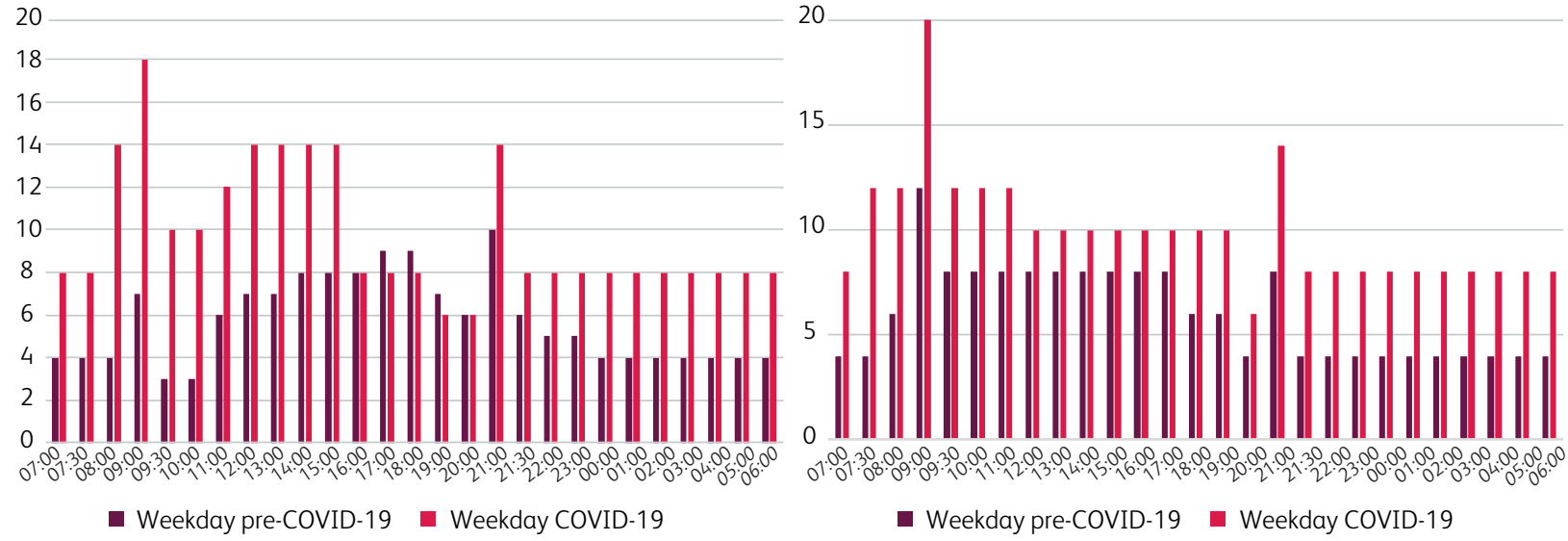

Fig 3. Comparison of SHO presence by hour and the number of senior house officers on the original rota and the COVID-19 rota during (a) weekdays and (b) weekends.

> It was agreed that core medical trainees with full MRCP qualification could 'act up' (sanctioned by Health Education West Midlands).

> Registrars no longer participated in ward specialty work, including referrals, which was taken over by the consultants.

$>$ Six individuals acted up as registrars from senior house officer (SHO) levels and eight registrars were moved from other nonGIM medical specialties.

> Seven respiratory registrars and 12 respiratory juniors were taken off the GIM on-call rota to provide a dedicated respiratory rota.

Rota for 'senior house officers' (FY2, CMTs, junior specialty doctors) (6 April 2020 - 4 August 2020)

Original rotas were different at the two sites (City and Sandwell Hospitals). On each site we had $26 \mathrm{SHO}$-level juniors, giving a total of 52 . While planning the COVID-19 rota, we calculated that 72
SHOs would be required. This was based on 60-bedded AMUs requiring five $\mathrm{SHO}$ in the day and four $\mathrm{SHO}$ at night. European Working Time Directive compliance was also ensured. This would give a 1 in 4 on-call (see Fig 3).

$12 \mathrm{SHOs}$ were redeployed to intensive care and, as mentioned above, six medical SHOs 'acted up' to registrar level. To create this rota, 38 SHOs were seconded from different specialities (including ophthalmology, obstetrics and gynaecology, paediatrics, cardiology, public health, acute care common stem and general practice) and the clinical teaching fellows were redeployed.

Rota for foundation year 1 (6 April 2020 - 4 August 2020)

The original foundation year 1 (FY1) medical rota had 14 FY1s at Sandwell Hospital and 17 FY1s at City Hospital. The rota was changed to have $20 \mathrm{FY} 1 \mathrm{~s}$ on each site ( 40 in total), supported primarily by FY1s seconded from surgical specialties (see Fig 4).

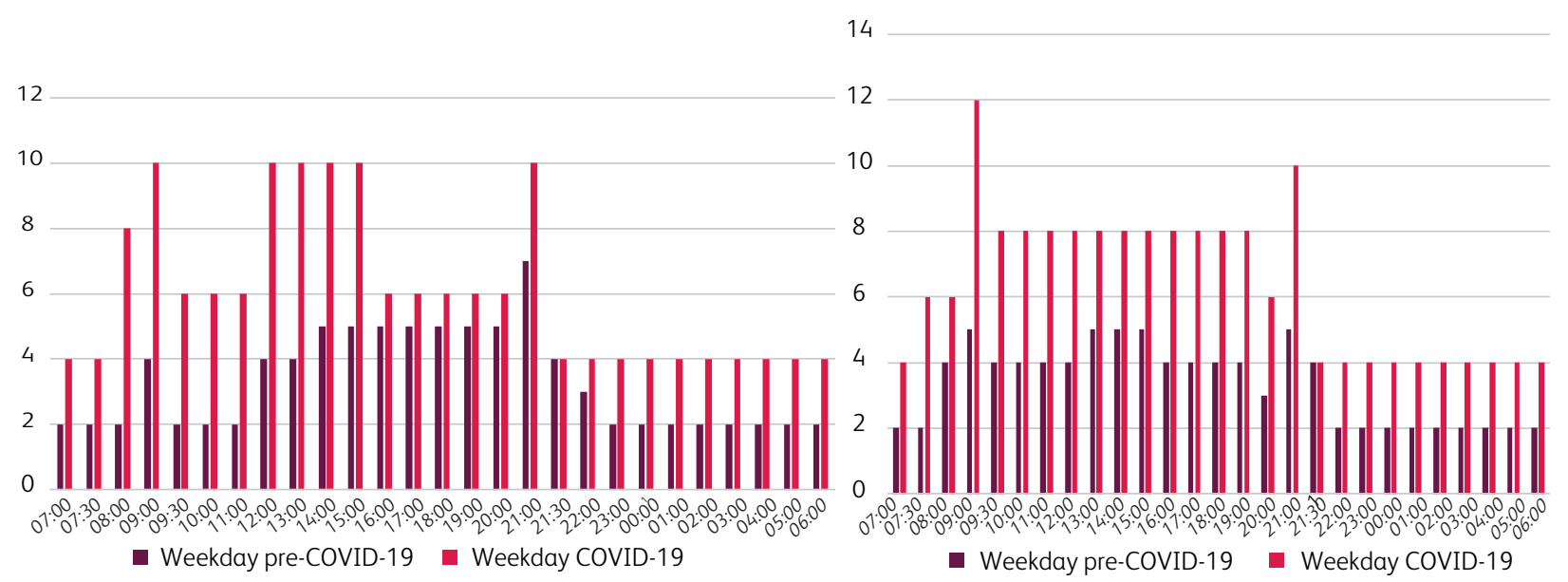

Fig 4. Comparison of FY2 presence by hour and the number of foundation year 2 doctors on the original rota and the COVID-19 rota during (a) weekdays and (b) weekends. 


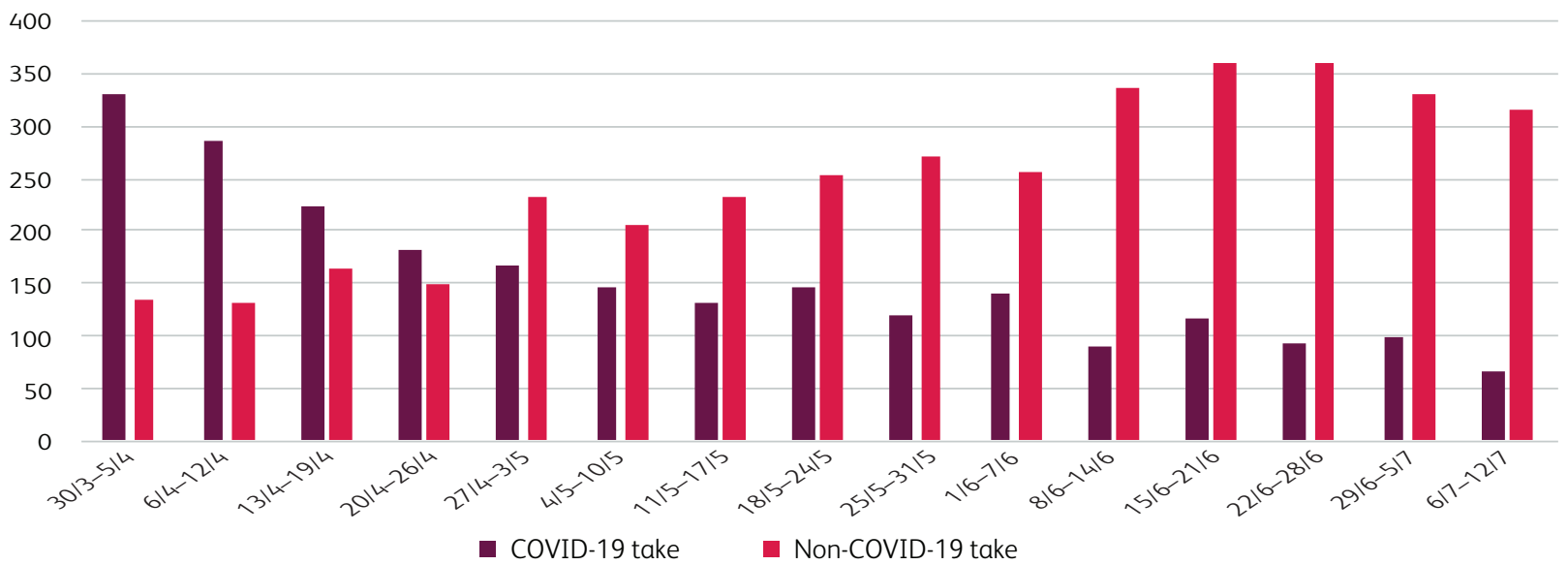

Fig 5. Weekly take totals and comparison between COVID and non-COVID medical takes between 30 March 2020 and 12 July 2020.

For all junior doctor grades, an induction pack and face-to-face induction was delivered by acute medicine and there was a regular review and visibility of consultants on the 'shop floor' to ensure trainees felt well supported.

\section{Results}

\section{Quantitative results}

Between 30 March 2020 and 12 July 2020 there were a total of 6,074 admissions. 2,340 (38.5\%) admissions were under the COVID take and $3,734(61.5 \%)$ non-COVID admissions. Fig 5 shows the weekly take totals between COVID and non-COVID medical takes. The COVID take peaked in week 1 and there was a steady decline thereafter. From week 5 (27 April 2020 to 3 May 2020) onwards, the majority of admissions were on the non-COVID take. Our average weekly takes across both sites ranges from 560-700 admissions. During the pandemic period, the average weekly take was 406 admissions (range 332-478 admissions).

Due to reconfiguration of the rotas we were able to increase the total number of working hours on the on-call rota. With respect to the registrar rota (COVID and non-COVID take) we were able to increase from 82.5 hours in a 24 -hour period during the weekdays to 122 hours ( $48 \%$ increase) on the COVID rota. During the weekends this was 101.5 hours pre-COVID which increased to 136 hours (34\% increase). For the SHO rota, the original rota had 137 working hours during weekdays and 142 working hours during weekends. This increased to 233 working hours ( $70 \%$ increase) and 231 working hours (63\% increase) respectively on the COVID rota. Finally, for the FY1 rota, the original rota had 78.5 working hours during weekdays and 79 working hours during weekends. This increased to 145 weekday working hours ( $85 \%$ increase) and 150 working hours on weekends ( $90 \%$ increase) on the COVID rota.

\section{Qualitative results}

Feedback from medical consultants was obtained via an email survey and informally via emails and face-to-face. 26 out of 48 (54\%) consultants responded to the formal survey which included 10 questions. $27 \%$ of the consultants who responded were not on the general medical rota beforehand.
$81 \%$ of consultants rated the induction training programme either good, very good or excellent and 19\% rated this average. Comments made by consultants included 'great real stories', 'clearer guidance on PPE required' and 'There were certain things - such as the Trust COVID information leaflet for patient discharges - that I wasn't aware of'.

$96 \%$ of consultants felt there was enough staffing on the oncall rota to cope with staff sickness, self-isolation and shielding. Comments from consultants varied from 'more than enough', 'very well managed' and 'Sometimes there was overstaffing during on-calls from a junior doctor [point of view].'

$100 \%$ of consultants said there was enough resilience on the medical on-call rota to cope with admission surges. Positive comments included 'SWBH medical on call rota is one of the best in the region not only for consultants but also for Spr and juniors' and 'To bring out a rota as complex and yet necessary within such a short time frame is a masterpiece.' One consultant called for 'more clarity regarding the exact working hours of the on-calls and how that related to clinical work. Those teams with permanent bed bases vs COW [consultant of the week] model working ended up with rotas with very different DCC [direct clinical care] due to lack of clarity'.

Another consultant commented on the workload and contribution of colleagues:

There is a need to have separate rotas for hot and cold takes. This is imperative. How we distribute the workforce will need to be decided on the number and type of patients we take in. On hindsight we did quite well. Those consultants who regularly do G(I)M seem to have got tired towards in the end. We must include more consultants.

Another consultant felt that the rotas should have been disbanded earlier due to the effect on outpatient and endoscopy waits.

$100 \%$ of consultants felt the separate COVID and non-COVID take streams worked well overall, although there were issues at the beginning with shared clinical spaces and with patients being wrongly moved to incorrect streams leading to complaints. One respondent suggested that when COVID numbers dropped there should have been some rebalancing of junior doctor deployment. $77 \%$ of consultants felt there was no drop in quality of care during the pandemic period on the acute medical take. Feedback 
included 'patients saw consultants promptly, we took out juniors clerking' and 'quality of acute medical take did drop with reduction in terms of not having the resources of quick imaging.' At times there was difficulty in switching patients "between streams from ED, especially if inappropriately put into hot stream'. Another consultant commented that the "drop was not as a result of the rota or local arrangements but rather as part of the wider challenge (NHS wide) in dealing with potentially high risk/highly infectious patients and the difficulties posed with assessments of presenting patients to the ED/AMU areas' and one consultant commented there may have been a potential drop in care for non-COVID patients, 'not forgetting that COVID patients also have other acute medical problems that need addressing. This can sometimes be missed as overshadowed by "COVID symptoms".

$92 \%$ of consultants reported that they felt supported by their consultant colleagues, especially over difficult cases. Feedback included 'we had a dedicated consultant who did Friday drop in counselling' and 'the acute physicians did the bulk of the work and ran the AMUs safely'.

$100 \%$ of consultants felt that the extra junior doctors on the rota helped improve efficiency over the running of the acute medical take. One consultant commented:

The COVID rota was a really impressive achievement. It does show how having adequately staffed rotas supports patient care and staff morale. Sadly it won't be possible to maintain this rota on a long-term basis, but it does show clearly that good junior doctor staffing has numerous benefits, and hopefully we can look to add additional doctors to rotas, either via additional trainees, or even a judicious and evaluated use of locum shifts to support areas/times where pressures tend to build up.

\section{Another commented:}

The take numbers were very small (in comparison to now and pre-COVID), and there were vast numbers of junior doctors. A lot of them with not very much to do. I think the take could have been just as efficient with fewer doctors during the day. BUT, if we have a second wave and the 'normal admissions' don't drop off, then we will need those masses of doctors to cope with the numbers of patients.

Another consultant agreed that having too many junior doctors at some points 'was probably better than too few' and 'good during the initial stages'. One consultant reported that 'if we had an excess [of junior doctors], we would often send them to the wards to help out. Often they returned as they were not needed.'

$26 \%$ of consultants felt inpatient care on general medical wards was affected during the pandemic period. One commented: 'Harder to get patients under the right speciality, so some gastro cases looked after by respiratory, chest cases looked after by endocrine etc. Also less consistency as consultants changed every few days. But overall these were not major problems.'

Consultants reported different experiences in different specialties. 'Respiratory provided an additional 24/7 telephone advice and additional weekend work, especially for the complex CPAP/NIV patients, without GIM commitment dropping.' A gastroenterology consultant felt that 'specialty care for gastro patients became very strained as our ward and patients were not protected, unlike other groups of patients/wards', while a consultant in stroke medicine commented that 'The stroke pathway was disrupted in many ways but I think that we managed to keep things together much better compared to other services'.
Qualitative feedback from consultants 'seconded' to the wards suggested that this was a positive experience for both groups; although the seconded consultants had some anxiety about being put into an alien environment, they felt welcomed and supported. Informal feedback from junior doctors was highly positive. A fifth-year medical student who joined acute medicine during the pandemic commented: 'It's been a great interim placement and a really supported introduction to working as a foundation doctor'. An ophthalmology SHO who was redeployed commented: 'It has been a great pleasure working with everyone on AMU. Thank you for looking after me for the last 3 months.' Finally, a medical registrar commented: 'I feel extremely lucky to work amongst such wonderful people and am really grateful to be working at this trust right now. It honestly really makes a difference to know that we have such an amazing and supportive team behind us, so thank you very much to all of you.'

\section{Discussion}

Redesigning rotas and creating new ones was a logistical challenge, especially under time pressure. We were able to learn and reflect from the new work plans. Having three working day shifts and twilight shifts allowed flexibility in the system and we were able to cover sickness relatively easily. We also found that there was no need for consultants to be resident on site overnight; all patients, in particular those on the COVID take, should have an appropriate escalation plan in place. Having a wide breadth of clinical expertise was a huge positive, with specialist knowledge and skills helping to provide high quality care for patients which in turn boosted morale.

Including other teams into general medicine has helped to support a 7-day working week model by allowing ward consultants breaks during the weekdays. The 7-day working model is an intense workload and has resulted in consultants working a 1 in 3 weekend which is not sustainable long-term. From the hospital admission data (Fig 5), we found a significant reduction in admissions on the COVID take and by the week ending 14 June 2020 this was $21 \%$ of all admissions. The consultant COVID rota ended on 15 June 2020 in view of the decline in COVID admissions sustained. In hindsight, we could have returned to the normal rota at an earlier date to reduce the impact on elective clinics and procedures.

We were pleased that feedback from non-ward-based specialty consultants was positive. They felt part of a team contributing to the pandemic; they could call for help and had access to a wide source of resources to help make clinical decisions.

The original plan of separate COVID and non-COVID emergency medical response teams (EMRTs) was not successful, as contact via switch led to confusion at times, and the Resuscitation team felt it was better to revert to the original system. In addition, not all non-COVID take registrars were Advanced Life Support compliant which meant running two separate teams was logistically difficult. Another difficulty that registrars met was diverting patients from COVID ED to non-COVID assessment areas.

A lot of planning and flexibility was required to create the rota for the $\mathrm{SHO}$ grade. All junior doctor rotas were compliant with the junior doctor contract ${ }^{2}$ and feedback from the juniors during this period was that they felt very well supported. It was felt to be important for junior doctors whose main post was in acute medicine to have breaks between COVID and non-COVID areas in order to prevent burnout and emotional injury. 
Prior to the COVID pandemic, there were significant problems relating to the number of patients being handed over from the day to the night team to the team (frequently $>10$ on both sites) to clerk. Current working patterns have resulted in significantly fewer numbers being handed over to the night teams (one or two at most), and as a result very few patients were handed over from the night to day teams. Rostered acute medicine juniors were used to supplement admission teams, resulting in a very short referral to review time.

Like the consultant on-call rota, the junior doctor rota is not sustainable long-term due to the heavy on-call commitment; booking annual leave was difficult. Both SHO and FY1 rotas would have preferred to have additional juniors for AMUs at weekends but in view of weekend frequency this is difficult to achieve (the junior doctor contract ${ }^{2}$ states that no rota should include more than 1:3 weekends).

In order to achieve <48-hour working week for the FY1 rota, a significant number of zero-hour days were rostered, affecting continuity and juniors' training and development. Ideally, we would have liked to roster more doctors at the weekend with shorter shifts, but a balance needs to be struck between frequency of weekend working and shift duration.

Juniors of all grades from non-medical backgrounds enjoyed their time on the acute medical take. They found the takes interesting and the wide range of pathology stimulating. In particular many commented that support they received from consultants was exemplary and they appreciated the good teamwork that was vital in these challenging times.

NHS England sent out an action letter on the 17 March 2020 asking all trusts nationally to postpone all non-urgent elective clinics and operations and to maximise staff availability to deal with the pandemic. ${ }^{3}$ The ongoing challenge is to restart urgent outpatient and procedural work while maintaining those changes felt to be beneficial and managing the ongoing COVID-19 and non-COVID-19 medical takes.

\section{Conclusion}

During this pandemic it has been a daunting challenge reconfiguring acute services and on-call patterns. We successfully implemented changes at short notice across each tier of medical professional group bringing in and supporting those unaccustomed with GIM working. We ensured high quality care for both doctors and patients. We aim to develop models of working that incorporates several of the changes we implemented. We hope this account of our urgent reconfiguration of services is of help to other trusts in the future.

\section{Acknowledgements}

To all the doctors who supported these changes in a selfless manner.

\section{References}

1 World Health Organization. Rolling updates on coronavirus disease (COVID-19). www.who.int/emergencies/diseases/novel-coronavirus-2019/events-as-they-happen.

2 NHS Employers. Terms and Conditions of Service for NHS Doctors and Dentists in Training England 2016. Version 4. NHS Employers, 2018.

3 Stevens S, Pritchard A. Important and urgent - next steps on NHS response to COVID-19. NHS England, 2020. www.england.nhs. uk/coronavirus/wp-content/uploads/sites/52/2020/03/urgentnext-steps-on-nhs-response-to-covid-19-letter-simon-stevens.pdf [Accessed 31 August 2020].

Address for correspondence: Dr Chetan Varma, City Hospital Birmingham, Sandwell and West Birmingham Hospital Trust, Dudley Road, Birmingham, B18 7QH.

Email: c.varma@nhs.net 